\title{
35. AN INVESTIGATION OF THE MOTION OF PERIODIC COMET BORRELLY FROM 1904 TO 1967
}

\author{
L. M. BELOUS \\ Northwestern Extramural Polytechnic Institute, Leningrad, U.S.S.R.
}

\begin{abstract}
The motion of $\mathbf{P} /$ Borrelly has been investigated using all precise published positions and taking into account the perturbations by Venus to Pluto. Nongravitational forces have been found to influence the motion of the comet considerably, formerly as a secular deceleration, more recently as a secular acceleration. By linking the seven apparitions 1904-1960, with allowance for the nongravitational effects, we have obtained a set of elements which represents 802 observations with a mean error of 1 ":89.
\end{abstract}

\section{Introduction}

Periodic comet Borrelly was discovered by Alphonse Borrelly at the Marseilles Observatory on 1904 December 28 as an object of magnitude 10, $1^{\prime}-2^{\prime}$ in diameter and possessing a small nucleus (Vsekhsvyatskij, 1958). Having a period of $6.9 \mathrm{yr}$, the comet was observed at eight apparitions 1904-1967; it was missed in 1939 and 1946. The first apparition of the comet was thoroughly investigated by Fayet (1914), who calculated a final orbit from 430 observations in eight normal places, taking into account perturbations by the six planets Mercury to Saturn; his set of elements represents the normal places with a mean error of 1".397. Later (Fayet, 1925), he formed ten normal places from 259 observations at the 1911-1912 apparition and obtained a set of elements that gave a good representation of the first nine normal places; the last normal place, formed from seven observations at the end of the apparition, produced a residual that was considerably larger than the rest. Fayet then linked the apparitions of 1904-1905 and 1911-1912 and derived a predicted set of elements for 1919, taking into account perturbations by the planets Venus, Earth, Jupiter, and Saturn.

\section{Investigation of the Comet's Motion under the Influence of Gravitational Forces}

We have investigated the motion of the comet from 1904 to 1967 , with the help of the BESM-4 computer at the Institute for Theoretical Astronomy. The following programmes have been used: integration with a variable step, in double precision and with allowance for perturbations by all the major planets (Kazimirchak-Polonskaya, 1967), integration by Cowell's method (Belyaev), comparison of observed and computed coordinates (Belyaev and Bokhan), and improvement of elliptical orbits (Bokhan and Makover). There were 105 accurate observations at the 1918-1919 apparition, 82 in 1925-1926, 33 in 1932-1933, 10 in 1954 and 7 in 1960. The 1967 apparition is represented by a single approximate observation. Using modern star catalogues, we have refined the positions of reference stars for the visual observations, proper motions being taken into account. 
We first considered the set of elements (A), obtained by Schaumasse (1931) from an integration of the motion from 1911 to 1919 , taking into account the perturbations by the planets Venus-Saturn:

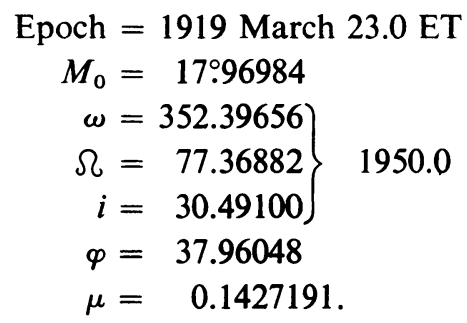

We have compared the 1918-1919 observations with the set (A) and concluded that these elements do not represent these observations adequately. We therefore made a preliminary orbit correction using 92 observations at the 1918-1919 apparition; normal places were not formed. The improved set of elements (I) represents the observations with a mean error of 2 ". 56 :

$$
\left.\begin{array}{rl}
\text { Epoch } & =1919 \text { March } 23.0 \text { ET } \\
M_{0} & =17.9742090 \\
\omega & =352.392113 \\
\Omega & =77.3686643 \\
i & =30.4899165
\end{array}\right\} \quad 1950.0
$$

The set (I) was adopted as a basis for further investigations. We integrated the motion up to 1925 and then up to 1932 , taking into account perturbations by all the major planets. We then succeeded in linking 87 observations at the returns of 1925 and 1932, obtaining the set of elements (II):

$$
\left.\begin{array}{rl}
\text { Epoch } & =1919 \text { March } 23.0 \mathrm{ET} \\
M_{0} & =17.9665203 \\
\omega & =352.391078 \\
\Omega & =77.3684744 \\
i & =30.4901315
\end{array}\right\} \quad 1950.0
$$

The mean error was 2".44. Using 38 observations at the same two returns Marsden (1968) obtained an orbit with mean error 2".5.

Our attempt to link the three apparitions 1918-1919, 1925-1926 and 1932-1933 gave a mean error of 15 ".4. On attempting to fit the observations at the 1932,1953 and 1960 returns Marsden found $(\mathrm{O}-\mathrm{C}$ ) residuals as high as 30". He pointed out that $\mathrm{P} /$ Borrelly evidently has a nongravitational secular acceleration, amounting to something like 0.05 per (period) ${ }^{2}$. 


\section{Influence of Nongravitational Forces on the Motion of the Comet}

On comparing the observations with the calculated orbit, and taking into account gravitational forces very completely, we confirm that $\mathrm{P} /$ Borrelly is affected by nongravitational forces, these appearing as systematic secular accelerations and decelerations, their values changing from apparition to apparition.

It is probable that this is not the only comet with this kind of motion. Instances where investigators have found only accelerations or only decelerations, or no nongravitational effects at all, can be explained by the small number of apparitions considered. It is possible that such variations in the action of the nongravitational forces could result in the failure to link particular apparitions of a comet, even when a large number of precise observations is available.

We have investigated the nongravitational variation of the mean anomaly $M_{0}$ of $\mathbf{P} /$ Borrelly during the whole period covered by observations. This investigation shows that from discovery to its approach to Jupiter (1936 March 26.04717, minimum separation $0.53935 \mathrm{AU}$ ) the comet had a secular deceleration; subsequently, the comet had a secular acceleration. The variations of $M_{0}$ and the corresponding variations in perihelion time $T$ are given in Table $\mathrm{I}$.

TABLE I

Nongravitational variations in mean anomaly $\left(\Delta M_{0}\right)$ and perihelion time $(\Delta T)$

\begin{tabular}{lll}
\hline Apparition & $\Delta M_{0}$ & $\Delta T$ \\
\hline 1911 & -0.0310 & +0.2180 \\
1918 & -0.0237 & +0.1660 \\
1925 & -0.0154 & +0.1076 \\
1932 & -0.0072 & +0.0503 \\
1953 & +0.0192 & -0.1366 \\
1960 & +0.0204 & -0.1453
\end{tabular}

\section{Improvement of the Elements}

The set of elements (III) was obtained by linking 120 observations of the comet at the five returns 1918-1960 and taking into account the variation of the mean anomaly due to the influence of nongravitational forces; the mean error was 1 ".61:

$$
\left.\begin{array}{rlrl}
\text { Epoch } & =1919 \text { March } 23.0 \mathrm{ET} \\
M_{0} & =17.9743901 \quad \pm 0.0000294 & \\
\omega & =352.3914940 \quad \pm 0.0001514 \\
\Omega & =77.3684353 \quad \pm 0.0000719 \\
i & =30.4900482 \pm 0.0000519
\end{array}\right\} \quad 1950.0
$$


Finally, we have incorporated the observations at the 1904-1905 and 1911-1912 apparitions, using for this purpose the normal places given by Fayet $(1914,1925)$, but omitting the tenth normal place at the second apparition. The set of elements (IV) satisfies the observations with a mean error of 1 ".89:

$$
\left.\begin{array}{rl}
\text { Epoch } & =1919 \text { March } 23.0 \mathrm{ET} \\
M_{0} & =17.9743786 \quad \pm 0.0000300 \\
\omega & =352.3915210 \quad \pm 0.0001439 \\
\Omega & =77.3684353 \quad \pm 0.0000752 \\
i & =30.4901341 \quad \pm 0.0000552
\end{array}\right\} \quad 1950.0
$$

The final elements for the seven apparitions considered are given in Table II. The set of elements for 1967 was obtained by using an approximate value for the nongravitational effects.

TABLE II

Improved elements (equinox 1950.0, times in ET)

\begin{tabular}{llccc}
\hline Epoch & 1905 May 15.0 & 1911 Oct. 31.0 & 1919 Mar. 23.0 & 1925 Aug. 3.0 \\
\hline$M_{0}$ & 16.8055948 & 353.101935 & 17.9743901 & 350.618223 \\
$\omega$ & 352.350961 & 352.373229 & 352.391494 & 352.424228 \\
$\Omega$ & 77.3815379 & 77.3758291 & 77.3684353 & 77.3785897 \\
$i$ & 30.4852542 & 30.4414967 & 30.4900482 & 30.5108143 \\
$\varphi$ & 37.9604383 & 37.8823025 & 37.9565787 & 38.0532219 \\
$\mu$ & 0.142776147 & 0.142261202 & 0.142752542 & 0.143173005 \\
$T$ & 1905 Jan. 17.2945 & 1911 Dec. 18.4893 & 1918 Nov. 17.0979 & 1925 Oct. 7.5250 \\
\hline
\end{tabular}

\begin{tabular}{llccc}
\hline Epoch & 1932 July 17.0 & 1953 Dec. 9.0 & 1960 Aug. 29.0 & 1967 Sept. 17.0 \\
\hline$M_{0}$ & 354.078527 & 25.6743858 & 10.7737019 & 12.8830691 \\
$\omega$ & 352.551043 & 350.948992 & 350.974719 & 351.035042 \\
$\Omega$ & 77.3083608 & 76.1780288 & 76.1942250 & 76.1406239 \\
$i$ & 30.5294665 & 31.0896158 & 31.0661465 & 31.1140951 \\
$\varphi$ & 38.0765135 & 37.1548362 & 37.1150968 & 37.1975837 \\
$\mu$ & 0.143401646 & 0.140679086 & 0.140381401 & 0.140862085 \\
$T$ & 1932 Aug. 27.2931 & 1953 June 9.5153 & 1960 June 13.2578 & 1967 June 17.5385 \\
\hline
\end{tabular}

\section{Acknowledgments}

It is my pleasant duty to express my cordial thanks to G. A. Chebotarev, Director of the Institute for Theoretical Astronomy, for the opportunity to carry out these calculations using the computer at the Institute. I also express my sincere thanks to E. I. Kazimirchak-Polonskaya for supplying her integration programme, for her helpful comments and constant attention to the investigation; I also thank N. A. 
Belyaev and N. A. Bokhan for their comments and for supplying computer programmes.

\section{References}

Fayet, G.: 1914, Ann. Obs. Paris Mem. 30, A1.

Fayet, G.: 1925, J. Obs. 8, 109.

Kazimirchak-Polonskaya, E. I.: 1967, Trudy Inst. Teor. Astron. 12, 39.

Marsden, B. G.: 1968, Astron. J. 73, 367.

Schaumasse, A.: 1931, J. Obs. 14, 147.

Vsekhsvyatskij, S. K.: 1958, Fizicheskie Kharakteristiki Komet, Moscow. 